\title{
Analysis of Different Space Time Block Codes with Linear Receiver for Various Modulation Technique
}

\author{
Khushbu Sethi \\ Rajasthan Technical University \\ 160 A, II B, sec-2, Vaishali, Ghaziabad
}

\author{
Kiran \\ Jaipur National University \\ F-111, Vashali Nagar, Jaipur
}

\begin{abstract}
Space-time coding is designed with two-dimensional signal matrices to be transmitted over a period of time from a number of antennas. The structure of the signal enables to exploit diversity in the spatial and temporal dimensions to obtain improved bit error performance and higher data rates without bandwidth expansion. So it is clear that transmit diversity plays an integral role in space-time code design. The objective of this paper is to provide the description of different type of space time block codes and to provide the performance analysis of these codes without channel knowledge at the transmitter with different schemes for four transmit and one receive antenna. Here a new space time block code is proposed and compared with other codes.
\end{abstract}

\section{General Terms}

Wireless communication, space time coding.

\section{Keywords}

MIMO systems, diversity, bit error rate, linear receivers, OSTBC, Q-OSTBC.

\section{INTRODUCTION}

One of the most significant developments of the last decade, that play a key role in realizing tremendous growth, is wireless communication using MIMO antenna architectures.

In wireless communication, one main challenge is the transmission over channels that experience time-variant multipath fading. Such detrimental effects in wireless fading channels can be combated using space -time block coding (STBC), an efficient transmit diversity scheme with exploitation of the diversity advantage of multi-antenna systems. Orthogonal space time block codes (OSTBCs) achieve full transmit diversity with a low (linear) decoding complexity. In case of multi-input multi-output (MIMO) communication systems with more than two transmit antennas, full-diversity rate-one O-STBCs do not exist. To overcome the low-rate constraints of OSTBCs, Quasiorthogonal space time block codes (QO-STBCs) have been proposed which achieve higher data rate but does not achieve full diversity and have a substantially higher decoding complexity than the OSTBCs.

Use of multiple antennas at the transmitter and receiver creates a multiple-input multiple-output (MIMO) channel that not only offers higher transmission rates, but it can also decreases error rates which improve the system's reliability and robustness to noise compared to single antenna systems. In addition to this, multiple antennas can also be utilized in order to combat co-channel interference.
At the receiver due to multipath fading, the receiver cannot correctly detect the transmitted signal unless some less attenuated replica of the signal is provided to the receiver. This technique is called diversity, which can be provided using temporal, frequency, polarization, and spatial resources [9].

\subsection{Diversity}

The main idea behind diversity is to provide different replicas of the transmitted signal to the receiver. If these different replicas fade independently, it is less probable to have all copies of the transmitted signal in deep fade simultaneously. Therefore, the receiver can reliably decode the transmitted signal using these received signals [10]. Diversity techniques can be implemented in different ways.

In time diversity different time slots are used to achieve diversity. In frequency diversity different frequency bands are used to achieve diversity. In space diversity multiple antennas are deployed at either the transmitting end or the receiving end to achieve diversity.

Space diversity can further be divided into receive and transmit diversity.

Receive diversity is achieved using multiple antennas on the receiving end of the communication link. The method of using multiple antennas on the receiving end has been in use for a number of years to improve the bit error rate (BER) performance. The basic idea is to have multiple signals with different degree of fading or different channel transfer function ' $h$ '. The signals are then appropriately combined with the help of diversity combining techniques.

Transmit diversity is achieved using multiple antennas on the transmitting end of the communication link. The transmit diversity is far more advantageous in comparison to the receive diversity. This is due to the fact that with transmit diversity, multiple antennas transmit delayed versions of a signal, creating frequency-selective fading, which is equalized at the receiver to provide diversity gain. A base station often serves hundreds to thousands of remote units. It is therefore more economical to add equipment to base stations rather than the remote units. For this reason, transmit diversity [1] schemes are very attractive. The transmit diversity is a modern phenomenon.

\subsection{Channel Coding}

Channel coding may also be used to provide diversity in the wireless channel $[3,9]$. Examples of practical channel codes include convolutional and block codes, trellis-coded modulation, multi-level coding, bit-interleaved coded modulation, as well as the recently discovered capacityapproaching coding schemes such as turbo and low-density 
parity check codes, and coded modulation techniques. The idea in space-time coding is to encode information both spatially and temporally and transmit the encoded sequence over multiple antenna elements using the same bandwidth. In wireless communications, channel coding schemes are usually combined with interleaving to achieve time diversity in an efficient manner [12].

\subsection{Focus and outline of the paper}

The objective of this paper is to provide the basics of space time block codes and propose a new scheme. This paper is organized as follows. In Section II, the model of space time block codes is described. In Section III, the different types of space time block codes and their property are presented. In section IV, the simulation result and performance comparison of different space time block codes with different schemes are given. In Section V, Some conclusions are offered. Although the list of references is not intended to be exhaustive, the cited papers (as well as the references) will serve as a good starting point for further reading.

\section{SPACE TIME BLOCK CODES}

Space-time block coding is a simple and effective means of achieving transmit diversity when other forms of diversity may be limited or non-existent and they can be decoded efficiently at the receiver by simple linear processing of the set of received signals.

\subsection{Transmission Model}

A wireless communication system with $N$ antennas at the base station and $\mathrm{M}$ antennas at the remote is considered. At each time slot $t$, signals, $\mathrm{C}_{\mathrm{t}}^{i} \quad i=1,2, \ldots N$ are transmitted simultaneously from the $N$ transmit antennas. The channel is assumed to be a flat fading channel and the path gain from transmit antenna $i$ to receive antenna $j$ is defined to be $\alpha_{\mathrm{ij}}$. The path gains are modeled as samples of independent complex Gaussian random variables with variance 0.5 per real dimension. This assumption can be relaxed without any change to the method of encoding and decoding [2]. The wireless channel is assumed to be quasi-static i.e. the path gains are constant over a frame of length and vary from one frame to another. At time $t$ the signal $\mathrm{r}_{\mathrm{t}}{ }^{j}$, received at antenna $j$, is given by

$$
\mathrm{r}_{\mathrm{t}}{ }^{j}=\sum_{\mathrm{i}=1}^{n} \alpha_{\mathrm{ij}} \mathrm{c}_{\mathrm{t}}{ }^{j}+\mathrm{n}_{\mathrm{t}}{ }^{j} \ldots . \text { (1) where the noise }
$$

samples $\mathrm{n}_{\mathrm{t}}{ }^{j}$ are independent samples of a zero-mean complex Gaussian random variable with variance $n /(2$ SNR $)$ per complex dimension. The average energy of the symbols transmitted from each antenna is normalized to be one, so that the average power of the received signal at each receive antenna is $n$ and the signal-to-noise ratio is SNR.

Assuming perfect channel state information is available, the receiver computes the decision metric

$$
\sum_{t} \sum_{j}^{n_{r}}\left|r_{t}^{j}-\sum_{i=1}^{n} \alpha_{i j} c_{t}^{j}+n_{t}^{j}\right|^{2}
$$

over all codeword

$$
c_{1}^{1} c_{1}^{2} \ldots c_{1}^{n} c_{2}^{1} c_{2}^{2} \ldots c_{2}^{n} c_{l}^{1} c_{l}^{2} \ldots c_{l}^{n}
$$

and decide in the favour of the codeword that minimizes the sum.

\section{TYPES OF SPACE TIME BLOCK CODES}

\subsection{Transmit Diversity with two antennas: The Alamouti Scheme}

The ALAMOUTI code is the first STBC that provides full diversity at full data rate for two transmit antennas [3]. The Alamouti scheme is a simple transmit diversity scheme suitable for two transmit antennas. Two symbols are considered at a time, say $x_{1}$ and $x_{2}$, and they are transmitted in two consecutive time slots. In the first time slot, $x_{1}$ is transmitted from the first antenna and $x_{2}$ is transmitted from the second one. In the second time slot, $-x_{2}^{*}$ is transmitted from the first antenna, while $x^{*}{ }_{1}$ is transmitted from the second antenna. The signals $x_{1}$ and $x_{2}$ are picked from an arbitrary $(M$-ary) constellation. Since two symbols are transmitted in two time slots, the overall transmission rate is 1 symbol per channel use, or $\log _{2} M$ bits per channel use.

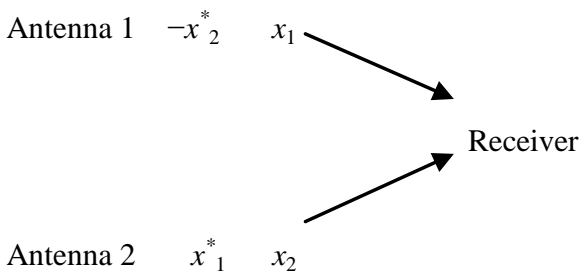

Fig 1: The Alamouti Scheme

\subsection{Orthogonal space time block code}

In this section, it is described the general space-time block codes based on the theory of orthogonal designs [5]. Consider a system with $N_{t}$ transmit antennas. The objective is to design a set of $N_{t} \times N_{t}$ matrices with elements from a desired signal constellation whose columns are orthogonal to each other. The latter property is required to make sure that a linear receiver is still optimal and the decoding complexity is still kept at a minimum. It is not always possible to find full-rate, full-diversity space-time block codes using (square) orthogonal designs. For real constellations, such a design is possible if the number of antennas is two, four or eight. If the square orthogonal design condition is relaxed, then it is possible to find other full-rate full-diversity code examples. On the other hand, for complex signal constellations (e.g., QAM, 8 PSK), full-rate designs exist if and only if $N_{t}=2$, that is, the Alamouti code is the only full-rate, full-diversity spacetime block code when the signal constellation is complex.

\subsection{Quasi-orthogonal space time block code}

The main characteristic of the codes designed in [5] is the orthogonality property of the codes. In Quasi orthogonal space time block code (JAFARKHANI code) [6], here propose structures that are not orthogonal designs and, therefore, at the decoder, cannot separate all transmitted symbols from each other. Instead, in Quasi OSTBC structure, the transmission matrix columns are divided into groups. While the columns within each group are not orthogonal to each other, different groups are orthogonal to each other. This is called as a quasi-orthogonal design. It is shown that using a quasi-orthogonal design, pairs of transmitted symbols can be decoded separately. It gives a way of obtaining full-rate (or 
increased-rate) space-time block coding designs using smaller designs as building blocks. The application of such a structure is in designing codes which provide higher transmission rates while sacrificing the full diversity. By using the orthogonality of the transmitted symbols, ALAMOUTI [3] first defined a space time transmission matrix as

$$
\mathrm{A}_{12}=\left[\begin{array}{cc}
X_{1} & X_{2} \\
-X_{2}^{*} & X_{1}^{*}
\end{array}\right]
$$

Where the subscript $A_{12}$ indicates the indeterminate $X_{1}$ and $X_{2}$ existing in the transmission matrix. Based on ALAMOUTI orthogonal STBC, JAFARKHANI [6] gave a quasi orthogonal STBC form for four transmit antennas as $\mathrm{C}_{J}=\left[\begin{array}{cc}A_{12} & A_{\mathrm{g} 4} \\ -A_{\mathrm{g} 4}^{*} & A_{12}^{4}\end{array}\right]=\left[\begin{array}{cccc}\mathrm{X}_{1} & \mathrm{X}_{2} & \mathrm{X}_{3} & \mathrm{X}_{4} \\ -\mathrm{X}_{2}^{8} & \mathrm{X}_{1}^{8} & -\mathrm{X}_{4}^{8} & \mathrm{X}_{3}^{8} \\ -\mathrm{X}_{3}^{8} & -\mathrm{X}_{4}^{8} & \mathrm{X}_{1}^{8} & \mathrm{X}_{2}^{8} \\ \mathrm{X}_{4} & -\mathrm{X}_{3} & -\mathrm{X}_{2} & \mathrm{X}_{1}\end{array}\right]$

Where $\mathrm{A}_{12}$ and $\mathrm{A}_{34}$ are ALAMOUTI codes. Further, different from JAFARKHANI scheme, the TBH case [7] has

$S_{A B B A}=\left[\begin{array}{ll}s_{12} & s_{\mathrm{g}} \\ s_{\mathrm{g}} & S_{12}\end{array}\right]=\left[\begin{array}{cccc}s_{1} & s_{2} & s_{3} & s_{4} \\ -s_{2}^{*} & s_{1}^{*} & -s_{4}^{*} & s_{3}^{*} \\ s_{3} & s_{4} & s_{1} & s_{2} \\ -s_{4}^{*} & s_{3}^{*} & -s_{2}^{*} & s_{1}^{*}\end{array}\right]$

Using a unitary pattern idea introduced in [8] to investigate the distribution of conjugates in the transmission matrices, it is finding that it is related to the positions of correlated values. By changing the distribution of conjugates, here matrices with different positions of correlated values can be obtained [11].

\subsubsection{JAFARKHANI Case with TBH Correlated Position}

Let the change conjugates' distribution of JAFARKHANI matrix, be

$$
C_{J T}^{H}=\left[\begin{array}{cccc}
x_{1} & x_{2} & x_{3} & x_{4} \\
-x_{2}^{*} & x_{1}^{*} & -x_{4}^{*} & x_{3}^{*} \\
x_{4} & -x_{3} & -x_{2} & x_{1} \\
-x_{3}^{*} & -x_{4}^{*} & x_{1}^{*} & x_{2}^{*}
\end{array}\right]
$$

\subsubsection{TBH case with JAFARKHANI- correlated positions}

Similar to the above modification, here the last row and the third row from eqn. (7) are exchanged and let

$$
C_{T j}^{H}=\left[\begin{array}{rrrr}
x_{1} & x_{2} & x_{3} & x_{4} \\
x_{2}^{*} & -x_{1}^{*} & x_{4}^{*} & -x_{3}^{*} \\
x_{4}^{*} & -x_{3}^{*} & x_{2}^{*} & -x_{1}^{*} \\
x_{3} & x_{4} & x_{1} & x_{2}
\end{array}\right]
$$

\subsection{Proposed Code}

Here a new space time block code matrix is proposed whose performance is better than other space time block codes. This space time block code is quasi-orthogonal in nature. Here zero-forcing technique is used for the analysis of this code. Channel is assumed to be quasi-static Rayleigh flat fading channel. The matrix of the proposed code is given by
$\mathrm{A}=\left[\begin{array}{ll}A_{12} & A_{94} \\ A_{34} & -A_{12}\end{array}\right]=$
$\left[\begin{array}{rrrr}x_{1} & x_{2} & x_{3} & x_{4} \\ -x_{2}^{*} & x_{1}^{*} & -x_{4}^{*} & x_{3}^{*} \\ x_{3} & x_{4} & -x_{1} & -x_{2} \\ -x_{4}^{*} & x_{3}^{*} & x_{2}^{*} & -x_{1}^{*}\end{array}\right]$

\section{SIMULATION RESULT AND PERFORMANCE COMPARISION}

In simulation result, first the comparison of ALAMOUTI space time block codes with $1 \times 1$ scheme is given. Then comparison with $1 \times 2 \mathrm{MRC}$ scheme is also provided. The comparison of analytical and simulation result is also given. ALAMOUTI scheme is better than other schemes but there is 3 - $\mathrm{dB}$ difference between ALAMOUTI scheme and $(1 \times 2)$ MRC scheme. Reason is that in ALAMOUTI scheme [3] the signal power is divided in 2 antennas equally.

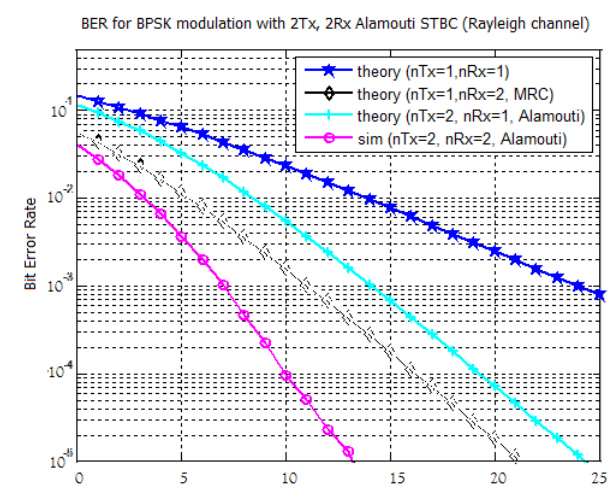

\section{FIG 1: BER performance of ALAMOUTI STBC [3] with different scheme}

In next results, comparisons of all the space time block codes explained in this paper are given. The codes are compared under the different modulation schemes like 8QAM, 16QAM, 32QAM, 64QAM, 128 QAM. It is seen that the proposed code has better performance than other codes under different modulation schemes. Linear receiver techniques like zero forcing are used in simulation model. Channel is assumed to be quasi-static flat fading Rayleigh channel.

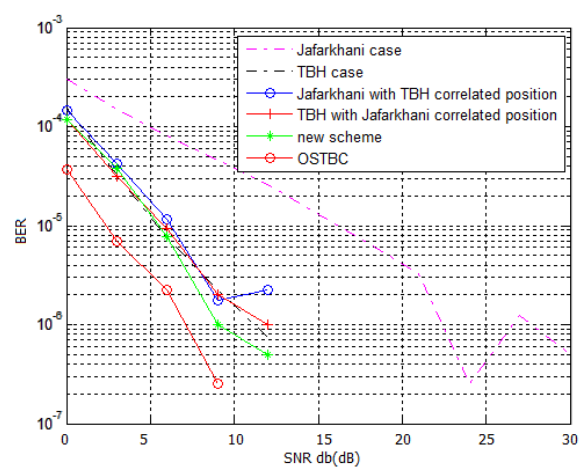

FIG 2: BER performance comparisons of different STBC under 8QAM scheme 


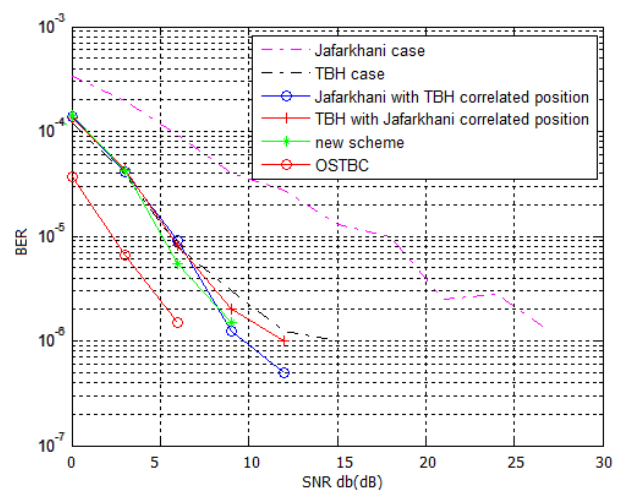

FIG 3: BER performance comparisons of different STBC under 16QAM scheme

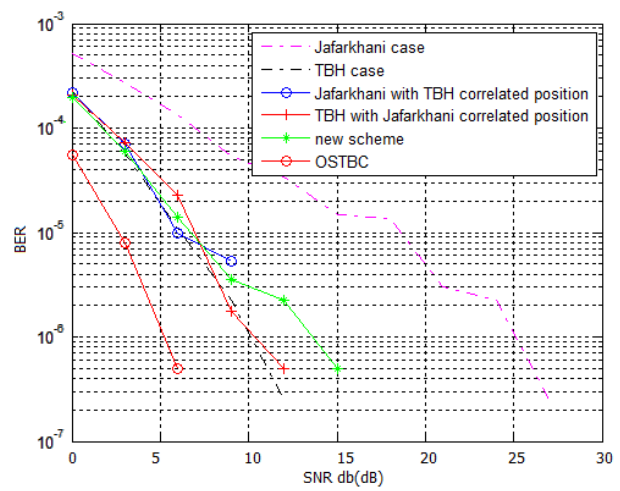

FIG 4: BER performance comparisons of different STBC under 32QAM scheme

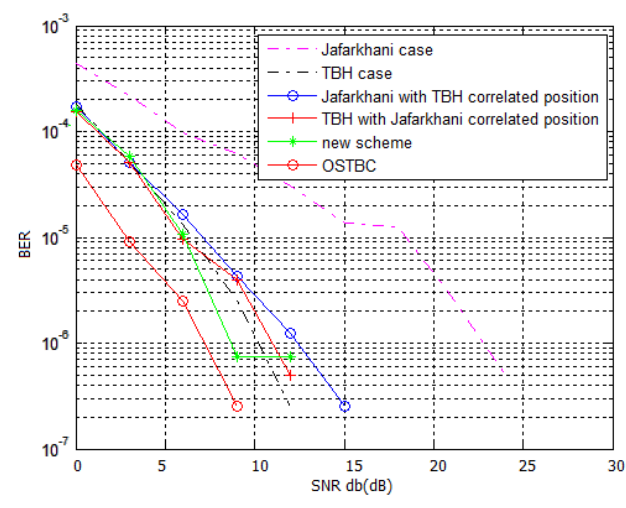

FIG 5: BER performance comparisons of different STBC under 64QAM scheme

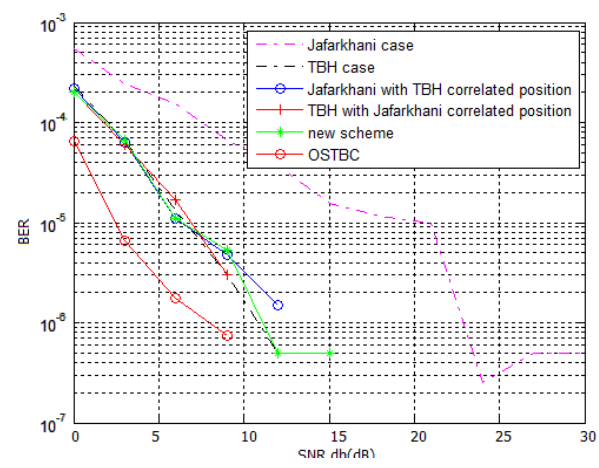

FIG 6: BER performance comparisons of different STBC under 128QAM scheme
Analyzing all the results, it is find that proposed code has better result than other space time block codes under linear receiver.

\section{CONCLUSION}

This paper describes the modeling of space time block codes. This paper shows comparison of different space time block codes to achieve better method of high diversity gain for different modulation techniques. For that different space time block codes (Jafarkhani, TBH, Jafarkhani correlated with TBH and TBH correlated with Jafarkhani, OSTBC) with their code matrix are explained and compared with proposed new scheme. Finally simulation results show that proposed scheme shows better results for QOSTBC in comparison to other QOSTBCs. There are still interesting open problems that deserve further investigation especially for $\mathrm{M} * \mathrm{~N}$ MIMO system (for $\mathrm{M}<1$ and $\mathrm{N}>1$ ).

\section{REFERENCES}

[1] J. H. Winters, "The Diversity Gain of Transmit Diversity in Wireless Systems with Rayleigh Fading," in IEEE Transactions On Vehicular Technology, Feb 1998.

[2] V. Tarokh, N. Seshadri, and A. R. Calderbank, "Spacetime codes for high data rate wireless communication: Performance criterion and code construction", IEEE Transcation on Information Theory, March 1998

[3] S.M. Alamouti, "A simple transmitter diversity scheme for wireless communications," IEEE J. Select. Areas Commun, Oct. 1998.

[4] J.Radon 1922 Lineare scharen orthogonaler matrizen, in Abhandlungen aus dem Mathematischen Seminar der Hamburgishen Universit"at.

[5] V. Tarokh, H. Jafarkhani and A. R. Calderbank, "Spacetime block codes from orthogonal designs,"IEEE Trans. Inform. Theory, July 1999.

[6] H. Jafarkhani, "A quasi-orthogonal Space time block codes," IEEE Trans. Commun., Jan. 2001.

[7] O. Tirkkonen, A. Boariu, and A. Hottinen, "Minimal nonorthogonality rate 1 space-time blok code for $3+\mathrm{Tx}$ antennas," in Proc IEEE 6th Int Symp. Spread-Spectrum Techniques and Applications (ISSSTA 2000)

[8] M.H. Lee, "A new reverse jacket transform and its fast algorithm," IEEE Trans. Commun., Jan. 2000.

[9] V. Tarokh, H. Jafarkhani, A. R. Calderbank "Space Time Bolck Coding for Wireless Communications: Performance Results" in IEEE journal on selected areas in communications, March 1999

[10] M. K. Simon, M. S. Alouini, 2005 Digital Communications over Fading Channels, Second Edition, Wiley Series in Telecommunications and Signal Processing,

[11] J. Hou, M. H. Lee, J. Y. Park "Matrices Analysis of Quasi-Orthogonal Space-Time Block Codes" in IEEE communications Aug 2003.

[12] J. Mietzner, R.Schober, L. Lampe, W. H. Gerstacker, P. A. Hoeher, "Multiple antenna techniques for wireless communications- A comprehensive literature survey" IEEE communications surveys \& tutorials, 2009. 